\title{
The effect of $\mathrm{LH}$ rise during artificial frozen-thawed embryo transfer (FET) cycles
}

\author{
Samer Khoury 1,2,*, Einav Kadour-Peero ${ }^{1,2, *}$ and Ilan Calderon ${ }^{1,2}$ \\ 1Division of Reproductive Endocrinology and Infertility, Department of Obstetrics and Gynecology, Bnai Zion Medical Center, Haifa, Israel \\ ${ }^{2}$ Technion-Israel Institute of Technology, The Ruth and Bruce Rappaport Faculty of Medicine, Haifa, Israel
}

Correspondence should be addressed to E Kadour-Peero: einavkadour@gmail.com

*(S Khoury and E Kadour-Peero contributed equally to this work)

\begin{abstract}
Purpose: To evaluate the association between a rise in serum luteinizing hormone (LH) levels during artificial frozenthawed embryo transfer (FET) cycles and clinical pregnancy rate.

Methods: A retrospective cohort study of women undergoing artificial FET cycles. We compared cycles in which LH double itself from the early follicular phase and further (group A) to cycles without a rise in LH (group B). Endometrium preparation was achieved by administration of $2 \mathrm{mg}$ three times per day estradiol valerate tablets. Embryo transfer (ET) was conducted after achieving endometrial thickness $>7 \mathrm{~mm}$ and vaginal progesterone was added according to the embryo's age. A beta-hCG was measured 13-14 days after ET. Clinical pregnancy was diagnosed on transvaginal ultrasound.

Results: Data from 984-FET cycles were retrieved. LH, exogenous estradiol (E2), progesterone values, endometrial thickness, and pregnancy outcomes were available in all patients. From 984-FET cycles, 629 (63.9\%) had a doubling, and $355(36.07 \%)$ had no rise in LH. Patients mean age was 30 years, similar in both groups. A multivariable logistic regression analysis was calculated to assess the effect of LH rise and pregnancy outcomes, after adjusting for confounders including a rise in $\mathrm{E} 2$ level and endometrial thickness. In this model, there was no association between doubling LH values and pregnancy rates (adjusted odds ratio: $1.06,95 \% \mathrm{Cl}: 0.75-1.5, P=0.74$ ).

Conclusion: LH rise during artificial FET cycles does not alter pregnancy rates. Apparently, hormonal monitoring of LH levels may not yield useful information in the artificial FET cycle and may be omitted.
\end{abstract}

\section{Lay summary}

Supplementation of estradiol, a hormone produced by the ovaries, starting at the beginning of the menstrual cycle of an artificially frozen embryo transfer (FET) can lead to a rise in luteinizing hormone (LH), the hormone that induces ovulation. Such a rise in LH may interfere with embryo implantation, the process where the embryo attaches to the inner lining of the uterus and, therefore, could affect the chances of pregnancy. The current study is the first to assess the effect of a dynamic rise in LH levels during FET cycles on pregnancy rates. This study found no difference in pregnancy rates between FET cycles where the LH doubled compared to cycles without such a rise in LH. Larger, prospective studies should be conducted to assess the impact of LH elevation on pregnancy outcomes.

Key Words: $>\mathrm{LH} \quad$ - clinical pregnancy $\quad$ artificial $\quad$ frozen embryo

Reproduction and Fertility (2021) 2 231-235

https://raf.bioscientifica.com

https://doi.org/10.1530/RAF-21-0017 (c) 2021 The authors Published by Bioscientifica Ltd

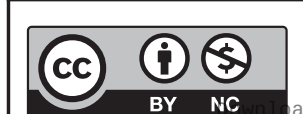

This work is licensed under a Creative Commons Attribution-NonCommercial 4.0 International License.

ded from Bioscientifica.com at 04/26/2023 08:34:30AM 


\section{Introduction}

Cryopreservation of embryos is a widely used procedure in assisted reproductive technology (ART) and has become a fundamental part of it (Gelbaya et al. 2006). Improved laboratory techniques and enhanced survival and implantation rates achieved by vitrification have contributed to the increasing trend for performing frozen-thawed embryo transfer (FET) cycles (Hakan 2016). The proportion of FET cycles in Europe reported by the ESHRE is about $32.3 \%$ (Kupka et al. 2011). As concordant with the European data, the proportion of FET cycles is $24.5 \%$ as reported by the most recent US nationwide database (CDC 2013). Performing FET in a spontaneous cycle may be favorable due to the fact that it mimics the natural process and eliminates adverse events of medication. A further advantage is a significant cost reduction compared to hormone therapy in artificial cycles (Hakan 2016). On the other hand, spontaneous FET may have disadvantages such as the timing of the procedure, which may be postponed due to anovulation in women with an irregular cycle, also, the timing of embryo transfer (ET) is not predicted and may cause higher rates of cancellation when compared with an artificial cycle (Gelbaya et al. 2006, Hancke 2012, Groenewoud 2017, Barbara 2020). Administration of exogenous estradiol (E2) and progesterone $(\mathrm{P})$ in artificial cycles may be favorable as it allows easy management and flexibility in the timing of the FET (Leeton 1991, Ben-Nun \& Shulman 1997, BalsPratsch 1999, Navot et al. 1989). Moreover, it has a lower cancellation rate (Ghobara \& Vandekerckhove 2008). Supplementation of E2 starting from the early follicular phase without pituitary suppression in an artificial FET can lead to a rise in LH levels, similar to that observed before ovulation in a natural cycle. Usually, E2 administration suppresses follicular recruitment and the LH rise, in these cases, it is not accompanied by ovulation and a rise in progesterone (Ziegler 1991, Simon 1998). However, such a rise of LH might interfere with the endometrial receptivity (El-Toukhy 2004) and, therefore, affects the pregnancy rate, a matter that has not been studied yet.

The aim of our study is to evaluate the association between an isolated elevating serum LH levels, in the absence of ovulation, during artificial FET cycles and pregnancy outcome.

\section{Materials and methods}

The present study was a retrospective single-center, clinical cohort study, which was approved by the local ethics committee. This study evaluated the data of all women undergoing artificial FET cycles, who attended the IVF unit of Bnai-Zion medical center in Israel between the years 2007 and 2016. Inclusion criteria were women undergoing FET in an artificial cycle with E2 supplementation. Exclusion criteria were stimulation protocols using lertozole, gonadotropins, gonadotropinreleasing hormone agonist (GnRH-a), -antagonist (GnRH-ant), and FET in the spontaneous cycle. Cycles in which a rise in progesterone levels was documented were also excluded.

All women eligible for artificial FET attended the Reproduction Unit on the second or the third day of their regular menstrual cycle where a transvaginal ultrasound was performed to exclude any ovarian cysts, and a blood sample for hormonal profile was obtained and included: E2, P, and LH levels.

During the artificial cycle, administration of estrofem (estradiol) or progynova (estradiol valerate) $2 \mathrm{mg}$ three times a day was started on the second or the third day of the natural menstrual cycle. Ten days after (average cycle day 12), a blood sample was withdrawn for hormonal profile testing that included E2, P, and LH, and transvaginal sonography (TVS) was performed to measure the endometrial thickness. The progestin drugs used were either utrogestan $200 \mathrm{mg}$ (micronized progesterone) or endometrin 100 mg administered vaginally three times a day and were started when the endometrial thickness reached at least $7 \mathrm{~mm}$.

The embryo transfer was carried out when all the above criteria were met and on the day of progesterone that matched the embryo's age. We compared cycles in which LH level increased (doubled or more) through the treatment cycle after E2 was started (group A) to cycles without a rise in LH levels or with a minimal rise that did not double the LH levels (group B). Endometrial thickness, E2, LH, P, and pregnancy outcomes were compared between the two groups. The transfer was conducted with the guidance of transabdominal ultrasound. The number of embryos transferred was according to the ASRM guidelines (Guidelines on Number of Embryos Transferred 2013). The quality of each embryo was graded according to The Istanbul consensus on embryo assessment (Istanbul Consensus Workshop 2011). A betaHCG was measured 13-14 days after ET. The primary outcome was clinical pregnancy, which was defined as fetal heartbeat on transvaginal ultrasound (Voluson S8, General Electric Corporation, USA) at 6-8 weeks of gestational age. 
Table 1 Demographic and FET cycle characteristics according to LH level in frozen-embryo transfer cycles $(n=984)$. Data are presented as $n(\%)$, median (range), or as mean \pm S.D.

\begin{tabular}{l}
\hline \\
Female age (years) \\
Gravidity ( $n$ ) \\
Transferred embryos ( $n$ ) \\
Embryo score (first embryo) \\
$\quad$ Good \\
$\quad$ Moderate \\
Poor \\
Embryo score (second embryo) \\
$\quad$ Good \\
$\quad$ Moderate \\
Poor \\
Embryo score (third embryo) \\
$\quad$ Good \\
$\quad$ Moderate \\
Poor \\
Endometrium (mm) \\
E2 (pg/mL) (CD 12) \\
Progesterone (nmol/L) (CD 12) \\
\hline
\end{tabular}

\begin{tabular}{c}
\hline Group A* \\
\hline 629 \\
$30.8 \pm 5.3$ \\
$1(0-9)$ \\
$2.3 \pm 0.7$ \\
$329(66 \%)$ \\
$137(28 \%)$ \\
$29(6 \%)$ \\
$239(50 \%)$ \\
$175(37 \%)$ \\
$60(13 \%)$ \\
$86(33 \%)$ \\
$90(35 \%)$ \\
$81(32 \%)$ \\
$9.3 \pm 1.7$ \\
$789(241-10,551)$ \\
$1.6(0.3-4.8)$
\end{tabular}

\begin{tabular}{c}
\hline Group B $\mathbf{~}^{\mathbf{1}}$ \\
\hline 355 \\
$31.4 \pm 5.1$ \\
$1(0-7)$ \\
$2.4 \pm 0.8$ \\
$180(66 \%)$ \\
$68(25 \%)$ \\
$23(8 \%)$ \\
$127(50 \%)$ \\
$92(36 \%)$ \\
$34(13 \%)$ \\
$53(36 \%)$ \\
$54(37 \%)$ \\
$40(27 \%)$ \\
$9.4 \pm 1.7$ \\
$689(95-9174)$ \\
$1.4(0.3-4.8)$ \\
\hline
\end{tabular}

\begin{tabular}{c}
\hline P value \\
\hline 0.076 \\
0.55 \\
0.13 \\
1 \\
\\
\\
0.1 \\
\\
\\
\\
0.66 \\
\\
\\
\\
\\
\\
\\
\\
\\
\\
\\
\end{tabular}

*Group A: with doubling LH; ${ }^{\dagger}$ Group B: without rise in LH.

$C D$, cycle day.

\section{Statistical analysis}

Statistical analysis was performed with SPSS 25.0 (IBM Corporation). The baseline population characteristics are presented as a mean and S.D. or as count and percentage. Data were evaluated for normal distribution using the Kolmogorov-Smirnov test. All continuous data were normally distributed. Demographics and pregnancy outcomes were compared using independent samples $t$-test or Fisher's exact tests as appropriate. $P<0.05$ was considered as significant. Multivariate Logistic regression model was assessed to determine the association between a rise in $\mathrm{LH}$ and pregnancy rate, after adjusting for rise in E2 level, and endometrial thickness above $7 \mathrm{~mm}$, on ET day.

\section{Results}

Data from 984 FET cycles of cleavage stage embryos were retrieved between the years 2007 and 2016. Progesterone, LH, E2 values, endometrial thickness, and pregnancy outcomes were available in all cycles. We compared the LH level in the early follicular phase to LH on the day in which the endometrial thickness reached the maximum and upon the ET day was appointed. From 984 FET cycles, 629 (63.9\%) had a doubling (group A), and 355 (36.07\%) had no rise in LH (group B). From those in which a rise in LH was documented, 366 (37.2\%) had tripling in LH levels. As mentioned, cycles in which a rise in progesterone levels was documented were also excluded. No significant clinical differences were observed between the two groups regarding women's age at oocyte retrieval day, gravidity, and cycle characteristics (number of embryos transferred, embryo score, E2, and progesterone levels) (Table 1). Mean women age was 30.8 years (range, 20-45 years) and was similar in the groups compared. Mean endometrial thickness was $9.3 \pm 1.7 \mathrm{~mm}$ in group A and $9.4 \pm 1.6 \mathrm{~mm}$ in group $\mathrm{B}, P=0.61$, Table 1 . Median serum levels of $\mathrm{LH}$ were statistically significantly higher in group A, $11.5 \mathrm{IU} / \mathrm{L}$ (7.3-16.0), and 7.4 IU/L (5.3-10.6), respectively $(P<0.001)$.

Biochemical pregnancy rate was similar in cycles with and without tripling LH level (25.4\% vs $24.4 \%$, respectively, $P=0.76$ ). No significant differences were present between the two groups with respect to clinical pregnancy rate, ectopic pregnancy, abortion and live birth rate $(20.3 \%$ vs $19.4 \%(P=0.8), 0.5 \%$ vs $0.3 \%(P=1.00)$, $7.2 \%$ vs $5.6 \%(P=0.42)$, and $13.2 \%$ vs $13.8 \%(P=0.84)$, respectively, Table 2 ).

Table 2 Pregnancy outcomes according to LH level.

\begin{tabular}{|c|c|c|c|}
\hline & Group A & Group B & $P$ value \\
\hline$n$ & 629 & 366 & \\
\hline Clinical pregnancy & $128(20.3 \%)$ & $69(19.4 \%)$ & 0.80 \\
\hline Live birth rate & $83(13.2 \%)$ & $49(13.8 \%)$ & 0.84 \\
\hline Abortion & $45(7.2 \%)$ & $20(5.6 \%)$ & 0.42 \\
\hline Ectopic pregnancy & $4(0.5 \%)$ & $1(0.3 \%)$ & 1.00 \\
\hline
\end{tabular}


A multivariable logistic regression analysis was calculated to assess the effect of LH rise and pregnancy outcomes, after adjusting for confounders including age, rise in E2 level, and endometrial thickness above $7 \mathrm{~mm}$, on ET day. In this model, there was no association between doubling LH values and clinical pregnancy rates (adjusted odds ratio: $1.06,95 \% \mathrm{CI}: 0.75-1.49, P=0.74)$.

\section{Discussion}

The present study addressed the question of whether the rise in endogenous LH levels can influence the likelihood of clinical pregnancy, in patients undergoing artificial FET cycle. The data presented herein show that the clinical pregnancy rate might not be affected by the rise in LH levels during artificial FET cycles.

Implantation is a dynamic process involving the apposition of a blastocyst-stage embryo to a receptive endometrium, attachment to the maternal endometrial epithelium, and invasion into the endometrial stroma (Loke 1995). Successful implantation is dependent on both embryological and endometrial factors, and embryos can only implant in a receptive endometrium (Psychoyos 1973). Previous study has shown that high LH levels may negatively affect endometrial receptivity and implantation since LH receptors are present in the endometrium (Ziecik 1992). However, according to our study, it might be assumed that endogenous LH levels probably have no direct effect on the endometrium and the process of implantation since that pregnancy rates were the same in both groups.

To date, two studies published have addressed the question about the association between pregnancy rate and the absolute value of $\mathrm{LH}$, the results of these studies are in line with those presented here, showing no association between absolute endogenous LH levels and the probability of clinical pregnancy (Cheung 2005, Griesinger 2007). One study by Cheung et al. showed that there was no difference in the absolute LH value, measured on day 14 of each cycle, between pregnant and non-pregnant women after artificial FET (Cheung 2005). The other study by Griesinger et al. was a prospective study that included 513 patients in their first FET cycle showed that there was no difference in pregnancy rate in cycles with low levels of $\mathrm{LH}$ (0.1-8.1 IU/L) compared to high levels (20-78 IU/L) measured 14 days after transdermal E2 patches (Griesinger 2007). This information, in addition to our results, might be useful in clinical practice since it indicates that dynamic changes in LH levels during the cycle or wide range of $\mathrm{LH}$ values is compatible with the establishment of clinical pregnancy, and thus there might be no value in measuring LH levels during artificial FET cycle.

Moreover, it is appealing to speculate that patients undergoing artificial FET as in the present study might not have benefited from pituitary suppression by a GnRH agonist. This is in line with a number of previous studies indicating no benefit of pituitary suppression in artificial FET cycles (Yee 1995, Simon 1998, Dal Prato 2002, Gelbaya et al. 2006, Griesinger 2007). Furthermore, it is important to note that the findings from the present study are only valid in artificial FET cycles but not in natural FET cycles or cycles with fresh embryo transfer following ovarian stimulation for IVF.

The main limitation of our study is its retrospective nature. However, our study was conducted in a single center with standardized protocols and the same management applied to every cycle. Moreover, our study represents one of the largest series addressing the association between LH rise in FET cycles and pregnancy rates.

\section{Conclusion}

Dynamic rise in LH levels during the FET cycle is compatible with the establishment of clinical pregnancy. We suggest that in artificial endometrium preparation cycles without pituitary down-regulation, the assessment of LH levels during E2 treatment may not appear to yield useful information regarding cycle management and patient prognosis. Therefore, hormonal monitoring of $\mathrm{LH}$ levels may be omitted. However, prospective randomized controlled trials should be conducted to confirm the results.

\section{Declaration of interest}

The authors declare that there is no conflict of interest that could be perceived as prejudicing the impartiality of the research reported.

\section{Funding}

This work did not receive any specific grant from any funding agency in the public, commercial, or not-for-profit sector.

\section{Ethical approval}

This research study was conducted retrospectively from data obtained for clinical purposes. The Intuitional Review Board reference is \#BNZ01001-16 (Bnai Zion Medical Centre).

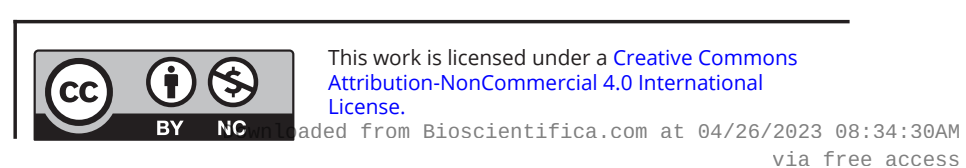




\section{Informed consent}

This retrospective chart review study involving human participants was in accordance with the ethical standards of the institutional and national research committee and with the 1964 Helsinki Declaration and its later amendments or comparable ethical standards.

\section{Consent to participate}

This is a retrospective study and patient consent was exempt.

\section{Author contribution statement}

E Kadour-Peero, protocol/project development, data collection and management, data analysis, manuscript writing/editing; S Khoury, manuscript conceptualization, manuscript writing/editing; I Calderon, manuscript writing/editing.

\section{References}

2013 Guidelines on number of embryos transferred. Fertility and Sterility 99 44-46. (https://doi.org/10.1016/j.fertnstert.2012.09.038)

Alpha Scientists in Reproductive Medicine and ESHRE Special Interest Group of Embryology 2011 The Istanbul consensus workshop on embryo assessment. Human Reproduction 26 1270-1283. (https://doi.org/10.1093/humrep/der037)

Bals-Pratsch M, Al-Hasani S, Schöpper B, Diedrich C, Hoepfner AS, Weiss J, Küpker W, Felberbaum R, Ortmann O, Bauer O, et al. 1999 A simple, inexpensive and effective artificial cycle with exogenous transdermal oestradiol and vaginal progesterone for the transfer of cryopreserved pronucleated human oocytes in women with normal cycles. Human Reproduction 14 (Supplement 1) 222-230. (https://doi.org/10.1093/humrep/14.suppl_1.222)

Barbara L, Carol C, Laura M \& Human MF 2020 The art of frozen embryo transfer: back to nature! Gynecological Endocrinology 36 479-483. (https://doi.org/10.1080/09513590.2020.1740918)

Ben-Nun I \& Shulman A 1997 Induction of artificial endometrial cycles with s.c. oestrogen implants and injectable progesterone in in-vitro fertilization treatment with donated oocytes: a preliminary report. Human Reproduction 12 2267-2270. (https://doi.org/10.1093/ humrep/12.10.2267)

CDC 2013 Assisted Reproductive Technology, National Summary Report. Available at (https://www.cdc.gov/art/pdf/2013-report/art_2013_ national_summary_report.pdf)

Cheung AP, Noble JM, Sierra S \& Wilson SJ 2005 LH and progesterone levels do not predict pregnancy success in cryopreserved embryo replacement using transdermal estradiol for endometrial synchronization. Human Reproduction 84 (Supplement 1) S172.

Dal Prato L, Borini A, Cattoli M, Bonu MA, Sciajno R \& Flamigni C 2002 Endometrial preparation for frozen-thawed embryo transfer with or without pretreatment with gonadotropin-releasing hormone agonist. Fertility and Sterility 77 956-960. (https://doi. org/10.1016/s0015-0282(02)02960-6)

El-Toukhy T, Taylor A, Khalaf Y, Al-Darazi K, Rowell P, Seed P \& Braude P 2004 Pituitary suppression in ultrasound-monitored frozen embryo replacement cycles: a randomised study. Human Reproduction 19 874-879. (https://doi.org/10.1093/humrep/deh183)

European IVF-Monitoring Consortium (EIM), European Society of Human Reproduction and Embryology (ESHRE), Kupka MS, D'Hooghe T, Ferraretti AP, de Mouzon J, Erb K, Castilla JA, Calhaz-Jorge C,
De Geyter CH, et al. Assisted reproductive technology in Europe, 2011: results generated from European registers by ESHRE. Human Reproduction 31 233-248. (https://doi.org/10.1093/humrep/dev319)

Gelbaya TA, Nardo LG, Hunter HR, Fitzgerald CT, Horne G, Pease EEH, Brison DR \& Lieberman BA 2006 Cryopreservedthawed embryo transfer in natural or down-regulated hormonally controlled cycles: a retrospective study. Fertility and Sterility $\mathbf{8 5}$ 603-609. (https://doi.org/10.1016/j.fertnstert.2005.09.015)

Ghobara T \& Vandekerckhove P 2008 Cycle regimens for frozenthawed embryo transfer. Cochrane Database of Systematic Reviews $\mathbf{1}$ CD003414. (https://doi.org/10.1002/14651858.CD003414.pub2)

Griesinger G, Weig M, Schroer A, Diedrich K \& Kolibianakis EM 2007 Mid-cycle serum levels of endogenous LH are not associated with the likelihood of pregnancy in artificial frozen-thawed embryo transfer cycles without pituitary suppression. Human Reproduction 22 2589-2593. (https://doi.org/10.1093/humrep/dem207)

Groenewoud ER, Cantineau AE, Kollen BJ, Macklon NS \& Cohlen BJ 2017 What is the optimal means of preparing the endometrium in frozen-thawed embryo transfer cycles? A systematic review and meta-analysis. Human Reproduction Update 23 255-261. (https://doi.org/10.1093/humupd/dmw046)

Hakan Y, Mehtap P, Sezcan M, Irem Y \& Gurkan B 2016 Preparation of endometrium for frozen embryo replacement cycles: a systematic review and meta-analysis. Journal of Assisted Reproduction and Genetics 33 1287-1304. (https://doi.org/10.1007/s10815-016-0787-0)

Hancke K, More S, Kreienberg R \& Weiss JM 2012 Patients undergoing frozen-thawed embryo transfer have similar live birth rates in spontaneous and artificial cycles. Journal of Assisted Reproduction and Genetics 29 403-407. (https://doi.org/10.1007/s10815-012-9724-z)

Leeton J, Rogers P, King C \& Healy D 1991 A comparison of pregnancy rates for 131 donor oocyte transfers using either a sequential or fixed regime of steroid replacement therapy. Human Reproduction 6 299-301. (https://doi.org/10.1093/oxfordjournals.humrep.a137326)

Loke YW, King A \& Burrows TD 1995 Decidua in human implantation. Human Reproduction 10 (Supplement 2) 14-21. (https://doi. org/10.1093/humrep/10.suppl_2.14)

Navot D, Anderson TL, Droesch K, Scott RT, Kreiner D \& Rosenwaks Z 1989 Hormonal manipulation of endometrial maturation. Journal of Clinical Endocrinology and Metabolism 68 801-807. (https://doi.org/10.1210/jcem-68-4-801)

Psychoyos A 1973 Hormonal control of ovoimplantation. Vitamins and Hormones 31 201-256. (https://doi.org/10.1016/s0083-6729(08)60999-1)

Simon A, Hurwitz A, Zentner BS, Bdolah Y \& Laufer N 1998 Transfer of frozen-thawed embryos in artificially prepared cycles with and without prior gonadotrophin-releasing hormone agonist suppression: a prospective randomized study. Human Reproduction $\mathbf{1 3}$ 2712-2717. (https://doi.org/10.1093/humrep/13.10.2712)

Yee B, Lin YP, Chacon RR, Soubra S, Rosen GF \& Cassidenti DL 1995 A simplified method of timing frozen embryo transfers. American Journal of Obstetrics and Gynecology 172 1844-1848. (https://doi. org/10.1016/0002-9378(95)91421-8)

Ziecik AJ, Derecka-Reszka K \& Rzucidło SJ 1992 Extragonadal gonadotropin receptors, their distribution and function. Journal of Physiology and Pharmacology 43 (Supplement 1) 33-49.

Ziegler D, Cornel C, Bergeron C, Hazout A, Bouchard P \& Frydman R 1991 Controlled preparation of the endometrium with exogenous estradiol and progesterone in women having functioning ovaries. Fertility and Sterility 56 851-855. (https://doi.org/10.1016/ s0015-0282(16)54654-8)

Received in final form 14 August 2021

Accepted 23 August 2021

Accepted Manuscript published online 23 August 2021 https://raf.bioscientifica.com

https://doi.org/10.1530/RAF-21-0017 (c) 2021 The authors Published by Bioscientifica Ltc

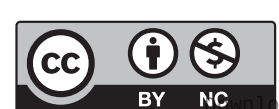

This work is licensed under a Creative Commons Attribution-NonCommercial 4.0 International License. ded from Bioscientifica,com at 04/26/2023 08:34:30AM 